\title{
Promoción de la salud en los lugares de trabajo: entre ideal e irreal
}

\author{
Health promotion in the workplace: between ideal and unreal
}

\author{
Alba Idaly Muñoz Sánchez \\ Universidad Nacional de Colombia. Sede Bogotá. Facultad de Enfermería \\ Eliana Castro Silva \\ Universidad Nacional de Colombia. Sede Palmira. Facultad de Ingeniería y Administración \\ Recibido: 30-06-10 \\ Aceptado: $21-10-10$

\section{Correspondencia:} \\ Alba ldaly Muñoz Sánchez \\ Universidad Nacional de Colombia \\ Torre de Enfermería Edificio 101 Oficina 711 \\ Carrera 30 N. ${ }^{\circ} 45-03$ \\ Bogotá D.C. - Colombia
}

\section{Resumen}

La Promoción de la Salud en los Lugares de Trabajo constituye una estrategia importante para abordar integralmente la salud y la seguridad en el trabajo, no obstante las actuales condiciones de precarización y flexibilización laboral interfieren con los principios promovidos por la estrategia.

Objetivo: describir la aplicación de principios como participación, justicia social, integralidad, sostenibilidad y multisectorialidad en la implementación de los programas de salud ocupacional en hospitales de tercer nivel de Bogotá, durante el segundo semestre de 2009.

Materiales y método: estudio de caso, descriptivo y transversal, basado en los principios que fundamentan la Promoción de la Salud, aplicando cuestionarios desarrollados para describir aspectos como política organizacional, recursos de apoyo, planificación, desarrollo y resultados alcanzados con la aplicación de la estrategia.

Resultados: las políticas definidas concuerdan con los propósitos de la estrategia analizada, pero, no hay coherencia con su aplicación en la práctica, pues se observan limitaciones en asignación de recursos, escasa participación del personal, falta de reconocimiento por la labor desempeñada, planificación tradicional de actividades y la desarticulación con otras instituciones del sector, entre otros.

Conclusiones: limitaciones conceptuales y de recursos para implementar la estrategia, al igual que el trabajo desarticulado de sectores como educación, empresas aseguradoras, etc.; conllevan al desaprovechamiento de mejores posibilidades para adecuar ambientes de trabajo.

Med Segur Trab (Internet) 2010; 56 (221): 288-305

Palabras clave (Mesh): Promoción de la salud / Lugar de trabajo / Salud Ocupacional / Sector de la salud.

Summary

Workplace Health Promotion is an important strategy to address holistically the health and safety at work, despite the conditions of precariousness and labor flexibility, hampering the principles advocated by the strategy.

Objective: to describe the application of principles such as participation, social justice, integrity, sustainability and multi-sectoral in the implementation of occupational health programs in third-level hospitals of Bogotá, during the second half of 2009. 
Materials and methods: a case study, descriptive and cross-sectional, based on the fundamental principles of Health Promotion, using questionnaires developed to describe aspects such as organizational policy, support resources, planning, implementation and results achieved with the implementation of the strategy.

Results: The defined policies are consistent with the purposes of the strategies analyzed; however there is no consistency in their application in practice as seen in resource allocation constraints, lack of staff participation, lack of recognition for the work performed, traditional planning and dismantling activities with other sector institutions, among others.

Conclusions: conceptual limitations and resources to implement the strategy, as disjointed work in sectors such as education, insurance companies, etc.; involves the wastage of better possibilities to adapt the work environment.

Med Segur Trab (Internet) 2010; 56 (221): 288-305

Key words: Health promotion / Workplace / Occupational Health / Health Care Sector. 


\section{INTRODUCCIÓN}

La salud y la seguridad en el trabajo, asumida desde una perspectiva amplia, involucra la comprensión y el análisis integral del ser humano, mirada ésta que implica reconocer condiciones individuales, intralaborales y extralaborales que, conjugadas, influyen en cualquier ambiente de desempeño de las personas. De acuerdo con esta visión, existe una propuesta denominada Promoción de la Salud en los Lugares de Trabajo-PSLT ${ }^{1,2}$ la cual, tras un amplio desarrollo conceptual, es entendida como el proceso integral y sistemático que sobre la base de una politica responsable y comprometida socialmente, establece un conjunto de acciones tendentes a crear condiciones para lograr una praxis que contribuya a fomentar valores positivos de la salud en los trabajadores; caracterizada además por su apertura a la participación, a la intersectorialidad y a la multidisciplinariedad que involucran múltiples acciones sociales contextualizadas, de manera que permita a los trabajadores incrementar el control sobre su salud para mejorar su calidad de vida, armonizando el proyecto de vida personal con el de la organización; por tanto, se revela como una herramienta de importancia estratégica para gestionar la salud y la seguridad en los ambientes laborales.

Por otra parte, las instituciones de salud son consideradas centros de gran importancia para implementar la PSLT ${ }^{3}$ por su potencial para abarcar problemas de salud desde una perspectiva integral y porque constituyen entornos significativos para las personas involucradas en el proceso del cuidado de la salud, representadas no solo en la fuerza de trabajo (personal de la salud), sino también en el objeto de trabajo que se explicita en los usuarios directos del servicio (pacientes) a través de los cuales se influye en otros grupos sociales (familiares y/o amigos).

Sin embargo, aparte de la variedad de factores de riesgo reconocidos en estos ambientes laborales ${ }^{4}$, es imposible obviar el hecho de que "en las últimas décadas se han introducido en América Latina y el Caribe una serie de reformas en el sector de la salud en las que las condiciones de trabajo no fueron incorporadas como prioridad" 5 y en consecuencia, se estructuran unas condiciones de precarización y flexibilización laboral que deben asumir estos trabajadores; razón por la cual se genera un serio cuestionamiento y a la vez constituye un reto, la forma de afrontar el mundo del trabajo en términos de salud y seguridad laboral en las instituciones de salud.

Por lo anterior, y observando las oportunidades y ventajas que ofrecen los centros de trabajo para promocionar la salud ${ }^{6,7}$, durante el segundo semestre del año 2009, se realizó una aproximación a la implementación de la estrategia en hospitales de tercer nivel adscritos a la Secretaría Distrital de Salud de Bogotá, buscando entender cómo se desarrolla la PSLT en estas instituciones.

Este estudio de caso con carácter descriptivo, partió de identificar las políticas aplicadas en el sector salud y sus implicaciones en la implementación de la estrategia; y se realizó con el propósito de aportar evidencia sobre la concepción y aplicación de la PSLT en instituciones hospitalarias y de contribuir a la búsqueda de mecanismos que permitan trascender de la teoría a la aplicación práctica, favoreciendo la calidad de vida de los trabajadores y de sus familias.

Así, este documento presenta los resultados obtenidos tras un acercamiento que implicó el análisis de la aplicación de los principios que fundamentan la PSLT, (participación, integralidad, justicia social, sostenibilidad y multisectorialidad e interdisciplinariedad) en el desarrollo de las actividades programadas a nivel organizacional, en cada caso seleccionado para estudio.

\section{MATERIALES Y MÉTODOS}

El proyecto se elaboró como un estudio holístico con múltiples casos ${ }^{8}$, de carácter descriptivo, transversal y con aplicación del método cuantitativo, cuyos criterios de 
inclusión permitieron identificar como universo de análisis a cinco unidades hospitalarias, del cual se seleccionaron tres casos participantes, los cuales, por consideraciones éticas, se identifican con las letras A, B y C.

Los instrumentos "ad hoc" para aplicar en el estudio, se definieron tomando como punto de partida los principios y las fases del proceso de la PSLT documentados y teniendo como referencia algunos estándares de calidad, guías, cuestionarios y otros formatos desarrollados por organizaciones como la Organización Mundial de la SaludOMS 3, 9, 10, la Organización Panamericana de la Salud -OPS 2,5_, la Organización Internacional del Trabajo -OIT ${ }^{11}$ - el Instituto Nacional de Seguridad e Higiene en el Trabajo-INSHT ${ }^{12}$ y otras comunidades interesadas en el tema ${ }^{13,14}$.

Para revisar y validar los instrumentos, se organizó un Pánel de expertos, en el que participaron docentes de varias universidades y un asesor y consultores de la OMS-OPS para Colombia, quienes realizaron importantes observaciones y aportes para continuar con el proceso. Posteriormente, se realizó una prueba piloto en una institución que cumplió con las mismas características de aquellas seleccionadas para el estudio, aplicándose los cuestionarios a quince trabajadores de todos los niveles; procedimiento que también arrojó observaciones importantes para ajustar definitivamente el instrumento a utilizar. Así, como se muestra en la tabla I, quedó establecido el instrumento y su operacionalización.

Tabla I. Manejo operacional de instrumentos en el estudio

\begin{tabular}{c|c|c}
\hline \multicolumn{1}{c|}{ Formato: } & \multicolumn{1}{c|}{ Para revisar: } & Diligenciado por: \\
\hline $\begin{array}{c}\text { Caracterización de la } \\
\text { población trabajado- } \\
\text { ra de la institución. }\end{array}$ & $\begin{array}{c}\text { La composición socio-demográfica y algunos aspec- } \\
\text { tos de la organización del trabajo. }\end{array}$ & $\begin{array}{c}\text { Responsable Oficina } \\
\text { de Talento Humano } \\
\text { y/o Salud Ocupa- } \\
\text { cional }\end{array}$ \\
\hline $\begin{array}{c}\text { Lista de chequeo: Pro- } \\
\text { moción de la Salud } \\
\text { en los Lugares de Tra- } \\
\text { bajo. }\end{array}$ & $\begin{array}{c}\text { El cumplimiento de algunos fundamentos de la PSLT } \\
\text { relacionados en información documental de la } \\
\text { institución. }\end{array}$ & $\begin{array}{c}\text { Investigador respon- } \\
\text { sable del proyecto. }\end{array}$ \\
\hline $\begin{array}{c}\text { Cuestionario: Nota Téc- } \\
\text { nica Preventiva NTP } \\
\text { 639* }\end{array}$ & El nivel de desarrollo de la PSLT, en la institución. & $\begin{array}{c}\text { Responsables de la } \\
\text { gestión relacionada } \\
\text { con salud y seguri- } \\
\text { dad en el trabajo. }\end{array}$ \\
\hline $\begin{array}{c}\text { Cuestionario: Mecanis- } \\
\text { mos de acción imple- } \\
\text { mentados. }\end{array}$ & $\begin{array}{c}\text { Los procedimientos e indicadores utilizados para ga- } \\
\text { rantizar participación, integralidad, intersectoriali- } \\
\text { dad en etapas de planeación, ejecución, resultados } \\
\text { y evaluación de acciones establecidas. }\end{array}$ & $\begin{array}{c}\text { Trabajadores en ge- } \\
\text { neral. }\end{array}$ \\
\hline $\begin{array}{c}\text { Cuestionario: Participa- } \\
\text { ción de trabajadores } \\
\text { en PSLT. }\end{array}$ & $\begin{array}{c}\text { El conocimiento y la participación de los trabajado- } \\
\text { res en las acciones ya mencionadas. }\end{array}$ & $\begin{array}{c}\text { Asesores de la ARP } \\
\text { asignados a la ins- } \\
\text { titución. }\end{array}$ \\
\hline $\begin{array}{c}\text { Cuestionario: Adminis- } \\
\text { tradora de Riesgos } \\
\text { Profesionales. }\end{array}$ & $\begin{array}{c}\text { El conocimiento y preparación de los asesores de las } \\
\text { ARP's en programas de PSLT. }\end{array}$ \\
\hline
\end{tabular}

* Definido por ENWHP y establecido como Nota Técnica Preventiva 639. Autorización de uso OT 374.09 dada por el INSHT para la aplicación en este estudio

La construcción de estos formatos y la preparación del trabajo de campo implicó también la elaboración de un documento para obtener el consentimiento informado de los trabajadores participantes en el estudio. En cumplimiento de los compromisos adquiridos con los participantes del estudio, previamente aprobado por el Comité de Ética de la Universidad Nacional de Colombia, el manejo de la información aseguró en todo caso las medidas necesarias para garantizar no solo la veracidad de la información suministrada sino también la confidencialidad de su origen.

Para la aplicación de los cuestionarios 3, 4 y 6, se definieron las personas involucradas en procesos de gestión relacionados con salud y seguridad en el trabajo de cada institución. Para estos casos, los formatos se aplicaron en forma censal (promedio de 10 personas por cada caso). Mientras tanto, para la aplicación del cuestionario 5, se consideró la inclusión y representación de todos los niveles y categorías de la población laboral, atendiendo el 
carácter integral que persigue la PSLT. Para este caso se calculó una muestra representativa de la población, utilizando el procedimiento para estimar proporción, que aplicó un error máximo admisible de 0,07 y un nivel de confianza de 0,95. Este resultado se ajustó definitivamente considerando el tamaño de la población trabajadora, quedando en promedio 170 personas para encuestar en cada caso. La selección de los participantes implicó la técnica de muestreo sistemático, tomando como marco muestral la nómina de trabajadores de cada institución. En este caso, la muestra incluyó proporcionalmente la participación de personal tanto de planta como de contrato.

La información recopilada a través de los cuestionarios se consolidó en una base de datos de Excel versión 2007 de acuerdo con las variables definidas en los instrumentos; los datos se analizaron utilizando técnicas para obtener frecuencias simples, desviaciones estándar y algunas correlaciones básicas. Aunque este análisis se hizo para cada caso en particular, se realizó una aproximación comparativa para reconocer patrones comunes y establecer conclusiones apropiadas.

\section{RESULTADOS}

Los hallazgos se analizaron teniendo en cuenta los criterios establecidos en la Nota Técnica Preventiva NTP 639 del INSHT, considerando la aplicación de los principios de la PSLT (ver gráfico 1).

Gráfico 1. Estructura de análisis de la información.

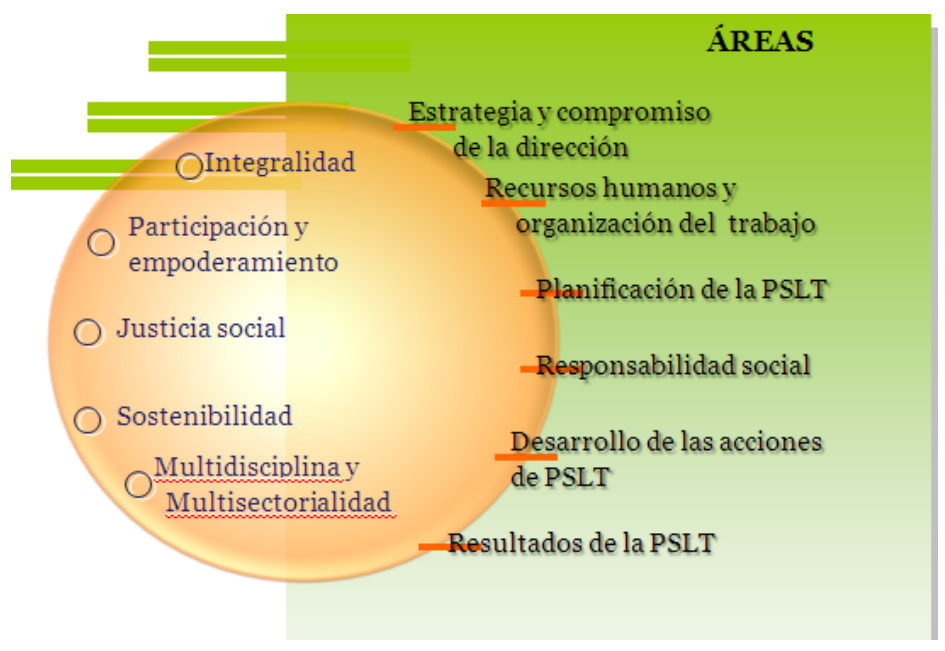

Con el propósito de discutir y justificar la expresión "entre ideal e irreal" como argumento principal de este escrito, se presentan algunos hallazgos obtenidos desde el referente documental, es decir, de lo manifestado textualmente por cada caso como políticas, programas y procedimientos, así como también, los resultados que reflejan las acciones implementadas en la práctica, desde la perspectiva de los trabajadores involucrados en estos ambientes laborales. Así las cosas, los resultados se relacionan con base en los principios de la PSLT ya mencionados.

El principio de participación que refiere la necesidad de brindar mayor seguridad y confianza a los trabajadores para tomar decisiones que impliquen su salud y desarrollar habilidades para promover iniciativas sobre el tema, constituye un principio corporativo que busca la integración del talento humano en la planeación, ejecución, evaluación y control de la gestión institucional y su responsabilidad en el éxito de la empresa; por lo tanto, se incluye como un deber de los funcionarios, reconociéndose así la intención de una participación abierta y motivada que involucre el proyecto de vida del trabajador con el proyecto del hospital. Dicha responsabilidad no está considerada para las etapas de formulación de políticas ni para planeación de acciones. 
Aunque oficialmente en Colombia se define el Comité Paritario de Salud OcupacionalCOPASO como medio para participar en el Programa de Salud Ocupacional-PSO solo el $22 \%$ de los trabajadores reportan haber utilizado este medio. Asimismo, un bajo porcentaje de trabajadores $(29,9 \%)$ confirma haber participado en estas actividades y tan solo el $8,5 \%$ considera que la institución ofrece algún tipo de incentivo a sus empleados para que aporten a la mejora de sus condiciones; esto último ratificado por el $75 \%$ de los responsables de la gestión involucrados en el estudio. Las razones manifestadas para justificar el bajo nivel de participación son: falta de tiempo, desconocimiento de las actividades, trabajo en el turno nocturno y el reiterado sentimiento de discriminación según el tipo de vinculación a la institución (planta \& contrato).

Sin duda, la importancia de la gestión del recurso humano que facilita la participación de los trabajadores, se ve notablemente afectada por la forma de contratación que aplican las instituciones, esto es, la vinculación de personal, sobre todo asistencial, por períodos máximos de un mes. Este sistema comporta una serie de condiciones que afectan no solo al equilibrio emocional del personal así contratado, sino que además constituye un factor de ineficiencia administrativa para la institución. De ahí que muchos empleados asimilan la vinculación por contrato como una "mordaza" que impide el ejercicio de la participación activa, teniendo en cuenta el temor que implica la inestabilidad laboral.

Por otra parte, no existe referencia a un mecanismo que promueva y facilite el proceso de retroalimentación con los trabajadores sobre los resultados obtenidos con las intervenciones realizadas.

El gráfico 2 presenta los resultados obtenidos al preguntar sobre la capacidad individual que cada trabajador considera tener, para participar en actividades como: notificar factores de riesgo, aportar ideas o sugerencias de mejora y hacer seguimiento a intervenciones implementadas. Al respecto, aunque los datos muestran que un alto porcentaje $(70 \%)$ se considera en capacidad de notificar factores de riesgo, también se evidencia la disminución de la capacidad sentida para participar en este tipo de procesos de mejora continua.

Gráfico 2. Capacidad reportada por trabajador para participar en actividades

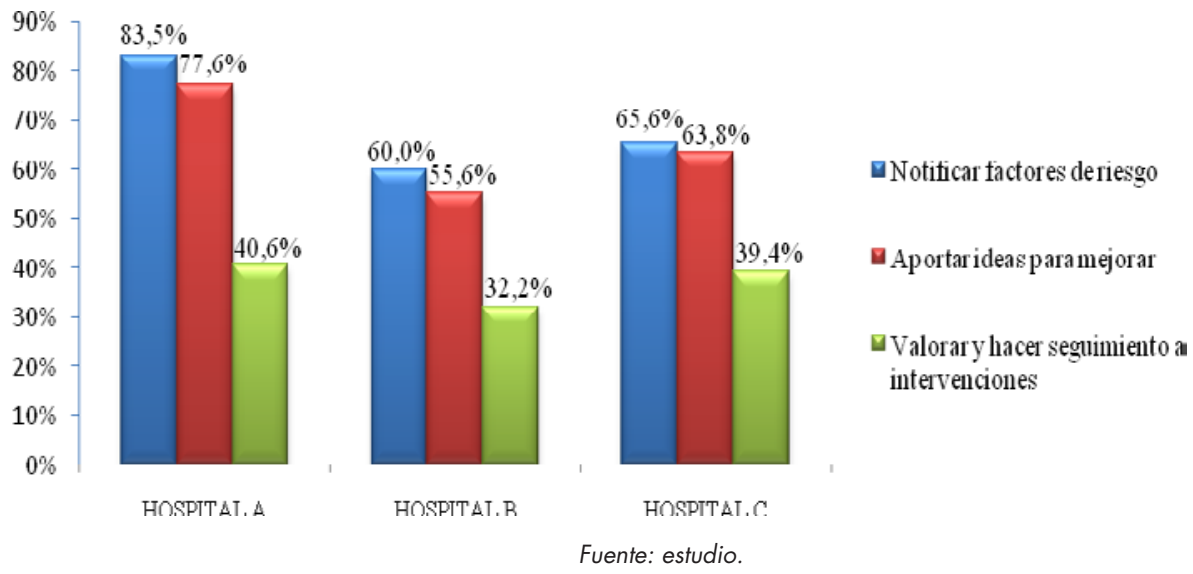

El principio de integralidad parte de entender las implicaciones de diversos factores (personales, ambientales, organizacionales, comunitarios e informativos) sobre el bienestar de los trabajadores e incorpora estrategias combinadas para lograr un rango de intervenciones que contribuyan con cambios de comportamiento en los individuos. Este principio tiene mucha relación con la forma de gestión del recurso humano y la organización del trabajo, lo cual involucra conocer al personal y considerar sus capacidades, limitaciones, experiencias y necesidades, de manera que dicho conocimiento se convierta en marco para fundamentar los procesos de planificación, desarrollo y evaluación de las actividades tendentes a la mejora integral del ambiente de trabajo. 
Aunque el conocimiento del personal comporta cierto grado de dificultad por la variabilidad de formas de vinculación establecidas en las instituciones, en teoría se observa la importancia dada a la recopilación y el análisis de datos sociodemográficos y de morbilidad, como soporte para el diagnóstico de condiciones de salud que analizadas junto a las condiciones de trabajo, estructuran el PSO, en cuya elaboración intervienen también otras dependencias encargadas de las acciones de bienestar social con el fin de orientarlo en forma integral, incluyendo además la opinión directa de los trabajadores sobre su salud. Pese a esto, ningún caso reportó información de los trabajadores discriminada según estado civil ni franja etaria, y tan solo en uno de ellos se hallaron datos relacionados con ocupación y máximo nivel de educación obtenido.

Adicionalmente, se definen procedimientos seguros para realizar la labor consignados en el manual de bioseguridad, concebido como una herramienta para incrementar la cultura de autocuidado mediante aplicación de normas universales; esta información se da a conocer a todos los trabajadores en los procesos de inducción y de capacitación periódica, lo cual es confirmado por el $54 \%$ de la población; pero, de este porcentaje el $25 \%$ niega su aplicación dentro de la labor, argumentando que no se proporcionan las condiciones necesarias para ejecutar dichos procedimientos. También se definen los Sistemas de Vigilancia Epidemiológica — SVE— para atender factores de riesgo de tipo psicosocial, biológico y ergonómico, entre otros.

Con frecuencia se refiere el desarrollo de actividades de educación, capacitación y entrenamiento en temas de salud ocupacional en las que priman aquellas relacionadas con factores de riesgo propios de la labor, buscando optimizar la calidad y la capacidad del talento humano. También se hace mención al fomento de estilos de vida y trabajo saludables, relaciones interpersonales, prevención de alteraciones cardiovasculares, prevención de tabaquismo, alcoholismo y drogadicción, prevención de cáncer de próstata y cérvix, prevención de la obesidad e hipertensión arterial, cuya implementación no fue confirmada por los trabajadores, durante el estudio.

Al respecto, los resultados sobre el desarrollo de programas dirigidos a trabajadores (ver gráfico 3) muestran un predominio del tema actividad física representado en charlas, pausas activas y programas de acondicionamiento físico, en respuesta al factor de riesgo ergonómico reconocido en este sector como uno de los principales causantes de accidentalidad laboral y enfermedad profesional. Aun cuando se mencionan planes complementarios de salud que incluyen programa de estilos de vida y trabajo saludables, en la práctica no se reconoció evidencia alguna, sobre el desarrollo de programas relacionados con salud reproductiva y sexual, nutrición, tabaquismo, alcoholismo o drogadicción.

Gráfico 3. Programas desarrollados en la institución, dirigidos a trabajadores

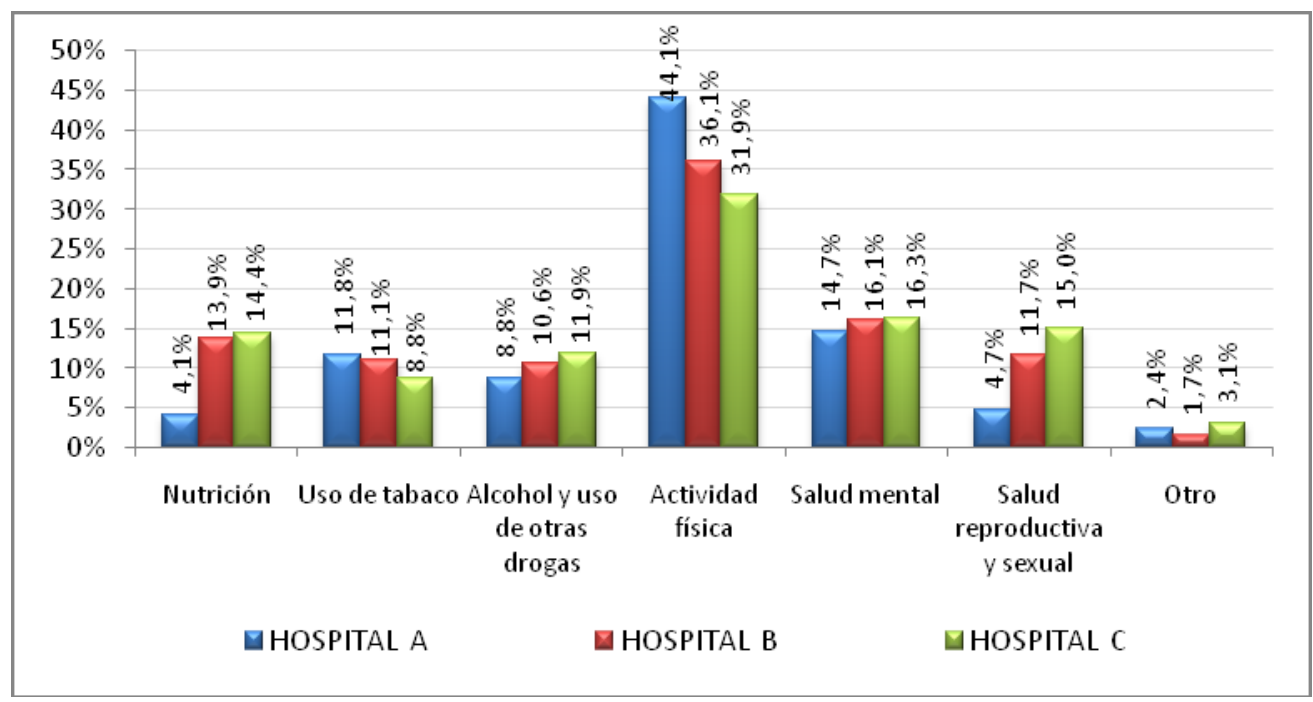

Fuente: estudio 
En suma, del 42,3\% de trabajadores que confirman el desarrollo de actividades de capacitación, educación y entrenamiento, tan solo el 29,8\% reconoce su participación en las mismas, argumentando que el bajo nivel de calidad percibida en estos procesos y el poco aporte personal y profesional que les significa, los lleva a reconsiderar la inversión del tiempo realizada para asistir a dichas actividades y, en consecuencia, al prejuicio con el cual se reciben propuestas de este estilo.

Los hallazgos sugieren que no se evalúan en forma integral y sistemática los resultados obtenidos con el desarrollo del programa, pues en principio, la evaluación solo incluye indicadores que se limitan a cuantificar proporciones y tasas referidas a enfermedad común, enfermedad profesional, accidentes de trabajo y ausentismo, los cuales abarcan un campo en extremo limitado para pretender valorar las acciones de programas enmarcados en la estrategia de la PSLT.

El principio de justicia social, que hace referencia al hecho de la inclusión total de las personas involucradas en los ambientes de trabajo, sin distinción de clase o rango, para efectos de cobertura en la aplicación de la estrategia de la PSLT, es contemplado en la política institucional a través de expresiones que evidencian el compromiso de mantener la calidad de vida y la integridad física y mental de trabajadores, contratistas, visitantes, estudiantes y personal de empresas en outsourcing que prestan servicios a la institución. Sin embargo, la normativa distrital ${ }^{15}$ establece que al contratar servicios externos (tercerizados) los hospitales públicos no tienen ninguna relación con el trabajador que se vincula para prestar el servicio, razón por la cual la institución no registra, ni analiza información para estos casos, como se pudo evidenciar en la fase de caracterización de la población trabajadora, lo cual constituye una forma de discriminación en los ambientes de trabajo, situación ampliamente percibida por la mayoría de los encuestados.

A pesar de esto, referencias como "la política cubre a todo el personal independientemente del tipo de vinculación que se establezca con la institución" se justifican si se tiene en cuenta que, de acuerdo con la legislación nacional ${ }^{16}$, las entidades estatales responden solidariamente por las acciones que derivan una responsabilidad civil, dentro del Sistema General de Riesgos Profesionales-SGRP. En consecuencia, la política organizacional destaca el cumplimiento de las exigencias legales por parte de todas las instituciones vinculadas a través de convenios docente-asistenciales y demás contratistas, considerando la responsabilidad solidaria para garantizar la seguridad y la salud individual y colectiva de los trabajadores.

Ahora, por definición estas instituciones juegan un papel significativo para la promoción de la salud tanto a nivel local como regional, por tanto, al tocar este principio es necesario abordar la responsabilidad social por medio de la cual se procura armonizar la calidad del servicio con las expectativas de la comunidad. Aspecto que se refleja en la política asistencial, la misión institucional, y en general, dentro del PSO. Por consiguiente, el contacto con la comunidad es estrecho a través de actividades vinculadas a programas de promoción y prevención, dirigidos a toda la población con el objeto de promover estilos de vida saludables y fomentar el auto cuidado con acciones de educación, información, concienciación e integración del individuo y la familia con los servicios de salud de la institución.

Igualmente, se encuentran definidos programas de gestión ambiental enfocados a la protección, manejo y cuidado del medio ambiente, cuyos principios y política incluyen ambientes sanos no solo para funcionarios y visitantes del hospital sino para la comunidad general. A pesar de las referencias que hacen de este aspecto algo tan evidente, resulta llamativo que aproximadamente el $61 \%$ de los trabajadores manifiesten no conocer si la institución participa en actividades con la comunidad de la localidad en la cual se ubica. Además, es muy poca la participación que se permite y aprovecha de los usuarios del servicio o personal de la localidad, en asuntos relacionados con salud y condiciones de trabajo de la institución, aspecto que solo fue confirmado por el 14,5\% de los trabajadores en general. 
El principio de sostenibilidad de la PSLT se garantiza cuando ésta hace parte integral de la gestión organizacional, en tal sentido, las instituciones establecen un compromiso a través de la Politica de Salud Ocupacional avalada por la Gerencia, en cuyo texto se resalta la importancia del tema para la organización y su interés por cuidar del recurso humano. Sin embargo, respecto de la asignación de recursos por parte de la institución para intervenciones en PSLT, llama la atención el contraste observado entre los hallazgos documentales y los datos reportados por quienes gestionan el proceso, pues este ítem resulta ser uno de los peor evaluados del componente "estrategia y compromiso de la dirección” de la NTP 639, alcanzando un 44,4\% de desarrollo; valoración ajustada si se consideran los escasos recursos tanto humanos, como físicos y financieros de que disponen las instituciones para llevar a cabo estos programas.

Por otra parte, el personal vinculado no solo ingresa mal preparado para afrontar los factores de riesgo propios de la ocupación, sino que además, por varios motivos un alto porcentaje (71\%) se muestra apático para participar en este tipo de actividades, situación que afecta el proceso de empoderamiento colectivo necesario para garantizar la sostenibilidad de la PSLT.

Sobre el principio de multisectorialidad e interdisciplinariedad, algunos documentos mencionan la necesidad de coordinar y asesorar con otras dependencias de la institución asuntos que involucren salud y bienestar de los trabajadores. Además, la existencia de varias políticas institucionales permiten deducir la existencia de otros grupos que apoyan actividades relacionadas con el tema, entre los cuales figuran el Comité paritario de salud ocupacional, el Comité de emergencias y brigada de emergencias, el Comité convivencia laboral, el Comité de personal, el Comité de epidemiología, el Comité de gestión ambiental y el Grupo especializado en mantenimiento preventivo y correctivo.

La conformación de cada grupo involucra, preferiblemente, personal de planta de la institución y para cada uno se encuentran definidos los términos de referencia con sus funciones. No obstante, su existencia no es divulgada a la totalidad de los trabajadores, tal como lo afirma el 60\% del personal de gestión y el $80 \%$ de los trabajadores encuestados, quienes manifiestan no conocer la existencia de dichos grupos. Además, no se tienen definidos indicadores o criterios que permitan evaluar el desempeño de los mismos.

Con relación a la existencia y vinculación de grupos externos a la institución, solo se referencian los asesores y contratistas de la Administradora de Riesgos Profesionales -ARP- quienes apoyan algunas actividades para intervenir factores de riesgo; apoyo éste que resulta insuficiente por la magnitud y complejidad de estas organizaciones.

A pesar de existir varias ARP vinculadas indirectamente a la institución a través del personal tercerizado (Vigilancia, Nutrición y Servicios generales), no hay información registrada que permita identificarlas, es decir, solo se reconoce aquella que oficialmente contrata la institución para proteger a sus empleados vinculados de planta, misma que por efectos de Ley ${ }^{17,18}$ también cobija a los de contrato; de tal manera, esta situación no permite aprovechar recursos comunes para desarrollar acciones sinérgicas y con mayor cobertura de la población trabajadora.

Por otro lado, se menciona el trabajo coordinado con el Ministerio de Protección Social -MPS—, la ARP, el Departamento Administrativo del Medio Ambiente -DAMA- y la Secretaría Distrital de Salud -SDS- sin embargo, esta relación se limita sobre todo a la vigilancia, control y rendición de cuentas sobre el cumplimiento de aspectos legales por parte de la institución. Situación que implica el constante reporte de informes y el consecuente desgaste del personal responsable de salud ocupacional.

En últimas, el gráfico 4 ilustra las respuestas dadas por los trabajadores con relación a si el programa de salud ocupacional da buenos resultados en la institución, datos que reflejan en cierta medida, la falta de confianza y el poco interés mostrado por la población trabajadora sobre las actividades que desarrolla el programa de salud ocupacional en la institución. 


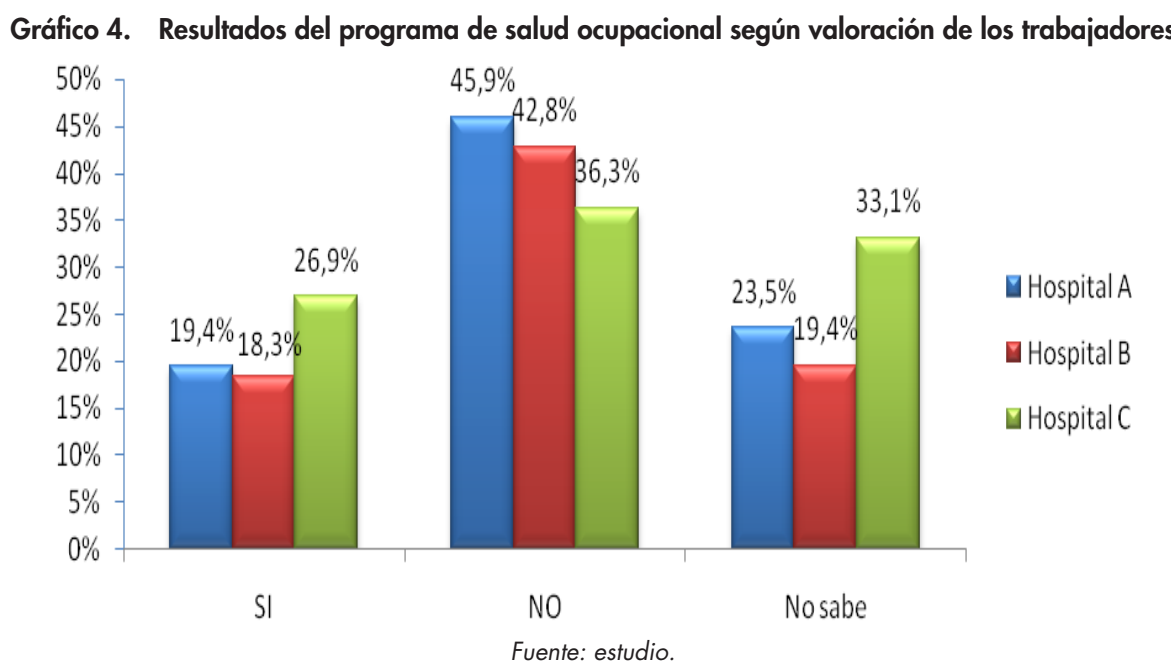

\section{DISCUSIÓN}

Para el caso estudiado, la directriz promocionada desde el ámbito internacional sobre políticas públicas saludables ha sido traducida en los niveles regional, nacional, distrital y de las mismas instituciones, dentro de las cuales es manifiesto el interés por vincular principios como participación, empoderamiento, integralidad, entre otros. Infortunadamente, en la mayoría de los casos revisados se observa que esto constituye un ideal, pues difícilmente se aplica en la práctica y no hay un claro interés por hacerlo efectivo. Esto podría pensarse, como un efecto indirecto de reformas que incidieron en el sistema de salud, como la implementada a finales de 1993, contexto en el cual "se ubicó la Promoción de la Salud como una función importante a ser desarrollada pero con un esquema organizativo que dividió tajantemente las acciones colectivas de las acciones individuales..." ${ }^{19}$, diluyendo funciones y responsabilidades sobre el tema en diferentes instituciones.

Ahora bien, "cuando se habla de promoción, se está en el territorio de la acción, de acciones coordinadas con una dirección y una finalidad" ${ }^{20}$, desafortunadamente, aunque en teoría mucha de la normativa formulada es consecuente con los fundamentos de la PSLT, con frecuencia en las expresiones particulares de actuación, la dimensión conceptual y las repercusiones de la estrategia no son claramente evidenciables, remarcándose la histórica confusión que existe entre los conceptos promoción de la salud y prevención de la enfermedad ${ }^{21}$, en el campo de la Salud y la Seguridad en el Trabajo. Adicionalmente, no se concretan procedimientos o mecanismos claros para su puesta en marcha, por consiguiente, en la práctica no se evidencian las acciones sugeridas en la Carta de Ottawa como crear ambientes de apoyo, establecer enlaces con la comunidad y desarrollar habilidades personales; definidas como recursos para lograr la implementación y sostenibilidad de la estrategia.

Ante este escenario, se destaca el papel y la responsabilidad de los que administran el cuidado de la salud, quienes a partir del compromiso y liderazgo deben involucrar a todos los trabajadores en el desarrollo, implementación y evaluación de políticas, procedimientos y programas demostrando la "capacidad constructiva de la organización" ${ }^{22}$ como elemento clave para la sostenibilidad de la estrategia de lugares de trabajo saludables, en lo posible, aplicando un enfoque socio-técnico que permita "un rediseño de procesos respetuoso con los valores y cultura de la organización, que promueva, mediante el uso de la tecnología una reestructuración del trabajo, mejorando las condiciones laborales, creando motivación e incrementando simultáneamente la eficiencia" 23. 
No obstante, los limitados recursos que se reservan para el funcionamiento de los planes de salud y seguridad en las instituciones afectan la capacidad de cumplir con el compromiso manifestado por parte de las altas directivas, para asegurar la puesta en marcha de la PSLT. Al respecto, algunos estudios sobre políticas saludables y su implementación en sectores públicos, privados y de voluntarios ${ }^{24}$, concluyen que nunca es suficiente la sola formulación de la política y que las principales barreras percibidas para su puesta en marcha radican en asuntos como la falta de recursos económicos y de tiempo, la falta de habilidades y experticia, desconocimiento de cuáles asuntos son prioritarios, entre otros. En consecuencia, se generan unas condiciones que desbordan la capacidad productiva de las personas encargadas y las posibilidades de respuesta de los programas formulados, conllevando reacciones de escepticismo hacia los programas y el consecuente desinterés por participar en las actividades relacionadas.

Por otra parte, las instituciones de salud "en los últimos años han estado inmersas en un proceso continuo de adaptación, fruto de fuertes presiones sociales y políticas, cuyo objetivo es mejorar los niveles de calidad asistencial y reducir los costes operativos" ${ }^{23}$, tal como puede evidenciarse en buena parte de la normativa colombiana relacionada $25,26,27$. Este panorama constituye una de las paradojas que hoy se enfrenta, relacionada con la certificación de la calidad del producto sin consideración por la calidad de las condiciones de trabajo de los involucrados en el proceso, aspecto que trae incomodidad entre los trabajadores, quienes viven el deterioro de las condiciones laborales y solo ven la preocupación de la institución al respecto, en procesos de habilitación o acreditación de la misma.

Estudios realizados sobre el sector ${ }^{28,29}$ muestran las desventajas que conlleva la forma de vinculación tercerizada, afectando la calidad del empleo y el clima laboral donde se prestan los servicios; más todavía, cuando no se percibe reconocimiento por la labor realizada ni valoración del mismo ${ }^{30}$, aspecto que fue reportado con un nivel muy bajo por parte de los trabajadores, con lo cual se generan sentimientos de inconformidad, rabia y desmotivación que predisponen negativamente a los trabajadores a participar y por tanto, disminuyen el espíritu de compromiso del personal con la institución; lo que en últimas redunda en detrimento de la calidad de vida de los mismos. Así lo refiere Barbosa de Oliveira ${ }^{31}$ en su estudio sobre el bienestar psíquico de los trabajadores de la salud en hospitales públicos, al destacar que las condiciones precarizadas de trabajo influyen no solo en la calidad de la asistencia sino que también trae perjuicios en la calidad de vida del trabajador.

Así las cosas, considerando las implicaciones del tema en la salud de los trabajadores, en marzo de 2009, la Alcaldía Mayor de Bogotá ${ }^{32}$ suspendió la contratación por intermedio de Cooperativas de Trabajo Asociado en el sector salud en Bogotá, basándose en las constantes quejas recibidas así como en estudios sobre el funcionamiento de esa modalidad realizados por la Secretaría Distrital de Salud; respuesta que surgió con el ánimo de garantizar la igualdad de condiciones de la población laboral del sector. En principio, estas acciones muestran las posibilidades relacionadas con la abogacía para promover la salud, cuando se cuenta con voluntad y compromiso político para llevarla a cabo.

Similar a lo observado con la política, documentalmente se destaca la importancia de vincular los principios de la PSLT en los procesos de planificación y ejecución de las actividades a nivel institucional, sin embargo es notoria la falta de claridad conceptual relacionada con el hecho de limitar la acción de la PSLT a un conjunto de actividades de educación, información y capacitación en salud ocupacional... ${ }^{33,34}$, y de evidenciarse además, una falta de interés por afrontar este tipo de discusiones conceptuales, por considerarse que poco aportan a mejorar la calidad de vida de los trabajadores ${ }^{35}$.

En general, mucho de lo implementado en términos de salud y seguridad en el trabajo en las instituciones comporta una respuesta forzada por el cumplimiento de la legislación en este campo, más que por un verdadero reconocimiento del valor que 
constituye el recurso humano vinculado, así, al afrontar los procesos desarrollados en las organizaciones y revisar la incorporación de los principios de la PSLT dentro de los mismos, se observa un tratamiento muy precario para todos ellos.

Por ejemplo, sobre el principio de integralidad que hace necesario por un lado, analizar la psicodinámica de la relación hombre-trabajo ${ }^{36}$ y por otro, caracterizar la población laboral buscando el reconocimiento de sus particularidades, teniendo como horizonte la formulación de actividades no individualizadas, sino que trasciendan a los colectivos buscando un impacto empoderador; es evidente el desconocimiento de mucha de esta información en los hospitales, lo cual muestra el limitado campo de acción que tienen los programas. En este sentido, las actividades planeadas y ejecutadas todavía hoy se reproducen en las mismas condiciones reportadas a finales de los noventa ${ }^{4}$, lo cual aduce la reproducción de un modelo tradicional con el cual se gestiona la salud ocupacional, que replica intervenciones con programas como los de acondicionamiento físico, de los cuales se ha observado que no logran buenos niveles de participación por parte del personal femenino ${ }^{37}$. No se encuentra el origen de la referencia, como efectivamente sucede en estas instituciones, donde además coexiste la necesidad de recursos necesarios para cumplir a cabalidad con los procedimientos establecidos para realizar el trabajo en forma segura.

Ahora bien, si se tiene en cuenta que "hoy el objeto social de las instituciones prestadoras de salud, ha migrado de uno cuyo propósito ha sido por tradición el servicio reactivo o curativo, a otro más proactivo, con una mirada integral que incluye a la comunidad, el entorno y los demás involucrados en el sistema, tal como lo plantea el enfoque estratégico" ${ }^{38}$ sobresale el hecho de que la seguridad del trabajador se procura por un período de tiempo y un espacio limitados a la jornada de trabajo, además del desconocimiento mostrado por el personal sobre acciones misionales de la institución como el servicio comunitario desarrollado a través de programas dirigidos a la población vulnerable de las localidades donde se prestan los servicios, lo cual indica dificultades relacionadas con el manejo de la información y el desconocimiento de los trabajadores sobre su empleador.

Además, para evidenciar la efectividad alcanzada por la gestión de riesgos profesionales, las referencias frecuentemente utilizadas se limitan a indicadores cuantitativos de accidentalidad laboral, enfermedad profesional y enfermedad general; en ningún caso se muestran valoraciones cuantitativas ni cualitativas relacionadas con efectos en productividad y mucho menos con períodos relacionados con el proceso de reproducción de los trabajadores, lo cual refleja el limitado enfoque de riesgos que caracteriza al sector y la consecuente visión reduccionista del trabajador como un individuo cuya vida se circunscribe al ambiente laboral.

Aun cuando se registra que una de las dificultades para el desarrollo de la PSLT es la falta de indicadores ${ }^{35,13}$ claros, precisos y apropiados para evidenciar los resultados alcanzados con su aplicación; en varios eventos realizados a nivel internacional y otras referencias documentales ${ }^{39}$ se presentan estudios de caso y experiencias contextualizadas en torno a la promoción de la salud los lugares de trabajo, que evidencian las ventajas que la estrategia conlleva mejorando el control de los factores de riesgo y aumentando los beneficios en la salud de la población involucrada, además de reducir tasas de ausentismo e incapacidades.

Frente a esta realidad, es importante considerar que los indicadores más allá de exponer apenas datos cuantitativos en términos de enfermedades y accidentes, deben contribuir a mostrar el desarrollo de diferentes etapas de los programas implementados tanto en estructura, como en procesos y resultados, que impliquen la capacidad laboral de las personas involucradas, de forma que se pueda tener una visión integral y continua del desempeño de los trabajadores. Al respecto, el estudio realizado, mostró la relevancia dada por el personal a preguntas que indagaron por el reconocimiento dado por la 
institución, a la motivación y a la satisfacción para desarrollar el trabajo, a la posibilidad de participación, entre otros.

El principio de participación condicionado por el nivel de empoderamiento de las personas, lo cual desde una perspectiva comunitaria como la que refiere Carvalho, posibilita el desarrollo de las capacidades propositivas, así como de habilidades para promover y proteger la salud en y desde los sitios de trabajo. Al respecto, Astolfo ${ }^{40}$ resalta la importancia de la capacidad transformadora del saber socialmente construido que fundamenta el diálogo social ${ }^{41}$, indispensable para afrontar situaciones como las de hoy, caracterizadas por factores que influencian en forma crítica la salud ${ }^{42,43}$. Por consiguiente, la participación se convierte en uno de los principios más reiterados a nivel documental en las instituciones estudiadas, a pesar de limitarse para las fases de ejecución, vigilancia $y$ control de las actividades desarrolladas, y excluirse para las etapas de formulación de políticas y de planeación del programa.

De cualquier forma, el estudio muestra una aproximación muy pobre a la práctica real del principio, entre otras cosas por la falta de procedimientos claros y de incentivos otorgados por la institución para hacer efectiva dicha participación. Adicionalmente, las mencionadas condiciones de contratación así como las de organización del trabajo, son reconocidas por los trabajadores como factores discriminatorios entre los trabajadores que imposibilitan la participación en los procesos institucionales.

También es importante analizar la postura que asumen los trabajadores frente al ejercicio de la participación, pues aunque se destacaron espacios como charlas, capacitaciones o reuniones de personal y la forma más utilizada para participar fue la de manifestar necesidades de tipo laboral, los resultados revelaron una falta de capacidad sentida para participar en sistemas que, como estos, involucran procesos de mejora continua. Una de las causas principales de este panorama es la falta de formación y de promoción de una cultura en salud y seguridad en el trabajo ${ }^{44}$, pues prima la idea de que estos situaciones solamente las manejan los que "saben" o "están encargados" del tema en la institución; es decir, que la responsabilidad frente al mismo es única y exclusiva de lo que se conoce como oficina de salud ocupacional y/o de su coordinador.

Tal como lo evidencian algunos estudios ${ }^{45}$ la participación de los trabajadores en este sector, no solo es relevante para mejorar las condiciones relacionadas directamente con su salud y su seguridad, sino también para atacar el potencial de accidentes y ocurrencia de errores que afectan al usuario en los servicios de salud. En efecto, es necesario reconocer el valioso potencial de aporte de los trabajadores en las instituciones, fundamentalmente, la experiencia en el desarrollo de la labor, la creatividad individual y las dinámicas de grupo internas pueden llevar al planteamiento de alternativas para mejorar o adecuar las condiciones de trabajo; es suficiente con hacerlo evidente a los trabajadores e incentivarlos para que sus iniciativas se traduzcan a la realidad.

Por otra parte, como se referencia en la Declaración de Sundsvall, el principio de multisectorialidad e interdisciplina cobra importancia cuando se trata de "fortalecer la defensa de la salud mediante la acción comunitaria, en particular a través de grupos organizados por mujeres, y se procura establecer alianzas a favor de la salud y ambientes favorables, a fin de fortalecer la cooperación entre campañas y estrategias sanitarias y del medio ambiente" ${ }^{7}$. Sobre el particular, se observa que aplica en forma muy limitada y si se quiere nula, pues a pesar que se establecen diversas políticas y grupos de apoyo para diferentes áreas de la institución y que desde una perspectiva integral podrían interactuar para lograr intervenciones de mayor impacto, las actuaciones responden a necesidades específicas de sus áreas de trabajo sin que se evidencie interés por compartir información entre ellos.

Además, en ningún caso se reconoció el establecimiento de alianzas estratégicas ni internas ni externas para buscar alternativas de actuación y de manejo de recursos. La única relación de apoyo y asesoría en salud y seguridad con una institución externa se establece con la ARP a la cual se afilia el hospital, de nuevo, por la responsabilidad 
legalmente asumida en la relación contractual. Con lo cual, se desaprovechan iniciativas interesantes generadas y promovidas individualmente por cada una de las instituciones y en consecuencia, se dificulta el ejercicio del benchmarking promovido y divulgado por la Red Europea de Hospitales Promotores de Salud.

A pesar de la referida situación respecto a la asignación de recursos para el desarrollo de estos programas que afecta al principio de sostenibilidad de la estrategia, se compensa considerando el interés que, por presión de tipo legal, permite que el programa exista cada año y se realicen algunas actividades en su nombre. De tal forma, la urgencia por mostrarse como una institución de calidad en los procesos de acreditación en los cuales se involucra la institución, no es bien recibida por los trabajadores por cuanto se entiende como una respuesta obligada a una directriz legal del nivel nacional y porque, adicionalmente, el interés mostrado por las directivas en dichos procesos no refleja la realidad contextual diaria de las instituciones.

En este sentido, se observa una relación inversamente proporcional entre el hecho de mantener en funcionamiento los programas de salud ocupacional por parte de las directivas de la institución y la credibilidad o confianza de los trabajadores en los mismos. Existe entonces la imperiosa necesidad de gestionar recursos para hacer más eficientes aquellos con los cuales se cuenta, esto tiene que ver con la organización de redes y/o grupos de apoyo intersectorial, así como la conjugación de recursos y actividades que vinculen otras instituciones que cubren al personal en la institución.

Es importante entender que no solo la disponibilidad de recursos físicos y financieros garantizan la implementación y sostenibilidad de la estrategia, también es importante el recurso humano, y en esta dimensión la fase de empoderamiento individual que facilitará a futuro una concreta participación colectiva, constituyendo y aprovechando el verdadero "valor universal de la democracia" ${ }^{46}$ comprendido desde la amplia perspectiva que presenta Amartya Sen, desde la cual "La participación social y política posee un valor intrínseco para la vida y el bienestar de los hombres".

Adicionalmente, siendo necesario para la sostenibilidad de la estrategia retomar la confianza de los trabajadores en estos procesos, es pertinente redefinir las dinámicas utilizadas para la inclusión y participación comprometida del personal en la mejora de las condiciones de trabajo, lo cual además involucra una mejora sustancial en los procesos de información, comunicación y registro de las actividades planificadas, así como de sus resultados. Dichas dinámicas pueden surgir de acciones propias de los grupos de trabajo a nivel institucional y ser socializadas y compartidas a nivel interinstitucional, o pueden ser propuestas evidenciadas en experiencias desarrolladas en otros contextos.

El principio de justicia social, igual que el de integralidad, se refleja documentalmente a través del PSO, el cual fija su interés en el cumplimiento de las necesidades y expectativas de todos los involucrados con la institución, bajo principios del Sistema de Garantía de la Calidad y Mejoramiento Continuo. No obstante, por las consideraciones de tipo organizacional referidas, este parece ser uno de los principios menos aplicados en los procesos institucionales revisados, pues la inclusión total del personal se complejiza teniendo en cuenta la variabilidad de la población involucrada y las condiciones de trabajo establecidas. En este sentido, variables como forma de vinculación, antigüedad, género, entre otras, puede traer efectos de estigmatización entre los grupos más diversos, trayendo menos satisfacción que grupos homogéneos ${ }^{47}$.

Adicionalmente, con la idea de priorizar la calidad del servicio sobre las condiciones laborales, se destaca la preferencia de la institución por atender en el proceso de trabajo, las observaciones, quejas y/o reclamos de los pacientes o familiares, no así para las situaciones o necesidades relacionadas con los trabajadores. Por consiguiente, no es de extrañar la evaluación otorgada por los trabajadores a los programas implementados, así como tampoco el hecho de que más de la mitad de los mismos consideren que el conocimiento que les brinda su institución sobre salud ocupacional, no les sirve para aplicarla en su vida cotidiana, con su familia o su comunidad. 


\section{Consideraciones finales:}

Abordar la revisión de los procesos desarrollados en los lugares de trabajo con el enfoque de la PSLT resulta coherente con la concepción holística que debe caracterizar el estudio de la salud y seguridad en el trabajo - -SST_- por medio del cual se entiendan los aspectos que componen el proceso de trabajo y se examinen las condiciones que contribuyen tanto con la productividad de las organizaciones como con los procesos de reproducción de la fuerza de trabajo. Esto, además, permite cimentar un pensamiento estructurado y un permanente cuestionamiento sobre las formas de actuar frente a las dinámicas cambiantes de los lugares de trabajo, buscando desplegar estrategias contextualizadas que respondan a las necesidades de los trabajadores y sus instituciones.

Aun cuando por la naturaleza de la metodología adoptada para la investigación, los resultados obtenidos aplican únicamente para los casos revisados y no admiten generalizaciones para el sector de la salud; el estudio permitió detallar un escenario laboral de relevancia social caracterizado por un soporte documental que, en forma ideal, subraya la importancia de implementar estrategias integrales como la PSLT al tiempo que, desde una perspectiva real y concreta, se destaca por presentar situaciones que obstaculizan su puesta en marcha, conllevando a que en la práctica no se evidencien avances significativos relacionados con estos procesos. Al respecto, es imposible pensar que se pueda crear o fomentar una cultura en salud y seguridad mientras las condiciones de trabajo no se adecuen para facilitarlo.

Ante este panorama, se puede dimensionar la difícil tarea que aguarda a todos los interesados en el tema para visualizar el escenario como una realidad, pero, al mismo tiempo constituye un reto para quienes, comprometidos por mejorar la calidad de vida de las personas, abordan la gestión del desarrollo productivo involucrando una perspectiva integral y buscando formas innovadoras para sortear las dificultades de estos tiempos. Al respecto, resulta interesante revisar experiencias que presenten y discutan ideas sobre mejores prácticas en el tema ${ }^{37,48}$ de manera que aplicando el benchmarking permitan desarrollar la capacidad de la comunidad y lograr el apoyo y la solidaridad necesarios para dinamizar la implementación de la promoción de la salud.

\section{BIBLIOGRAFIA}

1 Organización Mundial de la Salud. The Health-Promoting Workplace: Making it Happen. Geneva, 1998. Doc. WHO/HPR/HEP/98.9. p.1. (citado febrero 2009) disponible en la world wide web: http://whqlibdoc.who. int/hq/1998/WHO_HPR_HEP_98.9.pdf

2 Organización Mundial de la Salud-Organización Panamericana de la Salud. Estrategia de Promoción de la Salud en los Lugares de Trabajo (PSLT) en América Latina y el Caribe. San José de Costa Rica: Marzo, 2000. (citado marzo 2009) p.4. Disponible en la world wide web: http://www.cepis.ops-oms.org/bvsast/e/fulltext/ relcosta/relcosta.html.

3 World Health Organization. Regional Office for Europe. Implementing Health Promotion in Hospitals: Manual and self-assessment forms. Ed. Oliver Groene. Copenhagen: 2006. p.12 Disponible en: http://www. euro.who.int/document/E88584.pdf

4 Instituto de Seguros Sociales, Protección Laboral. Central Unitaria de Trabajadores -CUT-. Salud, Trabajo y Medio Ambiente en el Sector Salud. Bogotá: ISS, 1998. 87 p.

5 OPS. Salud y seguridad de los trabajadores del sector salud. Manual para gerentes y administradores. Washington, D.C. 2005. Cap. 3.

6 Comité de Expertos de la OMS. Fomento de la Salud en las Poblaciones Trabajadoras. Serie de informes técnicos. 765. Ginebra: 1988. (citado 10 marzo 2009) pp. 8-16. Disponible en la world wide web: http:// whqlibdoc.who.int/trs/WHO_TRS_765_spa.pdf

7 World Health Organization. Declaración de Sundsvall sobre los ambientes favorables a la salud. Sundsvall: 1991. Documento WHO/HED/92.1

8 Robert Stake. Investigación con estudios de casos. Ediciones Morata. Madrid: 1998. p. 17 
9 World Health Organization. Vienna Recommendations for Health Promoting Hospitals. (citado 10 marzo 2009). Disponible en la world wide web: http://www.euro.who.int/document//IHB/hphviennarecom. pdf

10 World Health Oragnization-Regional Office for the Western Pacific. Regional guidelines for the development of healthy workplaces. 1999. (citado 10 marzo 2009). Disponible en la world wide web: http://www.wpro. who.int/internet/resources.ashx/HSE/occupational_health/data/maa/Healthy+Workplaces+Guidelines.pdf. Consultado mayo de 2009

11 Organización Internacional del Trabajo. Directrices sobre sistemas de gestión de la seguridad y la salud en el trabajo. Op. cit. Disponible en: http://sobes.org.br/site/wp-content/uploads/2009/08/MEOSH-2001-2.pdf

12 Instituto Nacional de Seguridad e Higiene en el Trabajo. Nota Técnica Preventiva 639: La promoción de la salud en el trabajo: cuestionario para la evaluación de la calidad. Disponible en: http://www.insht.es/ InshtWeb/Contenidos/Estudios/Proyectos/En_Curso/Subvencionados/RedEuropeaPromoSalud/Ficheros/ ntp639.pdf

13 Riitta-Maija Hämäläinen. Workplace Health Promotion in Europe - the role of national health policies and strategies. Finnish Institute of Occupational Health. Editorial Vammalan Kirjapaino. 2007. Pp.73-74

14 Hans Martin Hasselhorn. Work Ability-Concept and Assessment. University of Wuppertal, Germany. 2008.

15 Alcaldía Mayor de Bogotá. Secretaría de Salud de Bogotá - Circular 001 de febrero 17 de 2009.

16 Colombia. Código Sustantivo del Trabajo. Art. 34 modificado por el artículo 3.․ del Decreto 2351 de 1965.

17 Ministerio de la Protección Social de Colombia. Decreto 2800 (2 octubre 2003). Art. 4. Por el cual se reglamenta parcialmente el literal b) del artículo 13 del Decreto-ley 1295 de 1994. Bogotá, D.C.: El Ministerio. 6 p.

18 Ministerio de la Protección Social de Colombia. Decreto 1931 (12 junio 2006). Por el cual se establecen las fechas de obligatoriedad del uso de la planilla integrada de liquidación de aportes y se modifica parcialmente el Decreto 1645 de 2005. Bogotá, D.C.: El Ministerio. 9 p.

19 Juan Carlos Eslava. Promoción y prevención en el sistema de salud en Colombia. Revista de Salud Pública. (on line). 2002; Vol. 4 n. 1 (citado 5 marzo 2009) p. 01-12. Disponible en la world wide web: http://www. scielo.org.co/scielo.php?script=sci_arttext\&pid=S0124-00642002000100001\&lng=en

20 Franco, D. Ochoa, M. Hernández. La promoción de la salud y la seguridad social. Foros sobre promoción de la salud en la seguridad social. Bogotá; Instituto de Seguros Sociales. Corporación Salud y Desarrollo. Julio 7 de 1995 , p. 6

21 Juan Carlos Eslava. Repensando la promoción de la salud en el sistema general de seguridad social en salud Rev salud pública. (on line) 2006; vol.8 n. ${ }^{\circ}$ 2. (citado 7 octubre 2009) pp.106 - 115. Disponible en la world wide web: www.scielo.org/pdf/rsap/v8s2a09.pdf

22 Dean Whitehead. Workplace health promotion: the role and responsibility of health care managers. Journal of Nursing Management, 2006; vol.14. n. ${ }^{\circ}$. (citado 15 marzo 2010) pp. 59-68. Disponible en la world wide web:http://www.bases.unal.edu.co:2109/login.aspx?direct=true\&db=a9h\&AN=19091220\&lang=es\&site=eh ost-live

23 Javier Osorio, Elsa Paredes. Reingeniería de procesos en los hospitales públicos: ¿Reinventando la rueda?. Revista Española de Salud Publica. (on line). 2001; vol.75 n. ${ }^{\circ}$ 3. (citado 2010-02-17), p. 204. Disponible en la world wide web: http://scielo.isciii.es/scielo.php?script=sci_arttext\&pid=S1135$57272001000300004 \& \operatorname{lng}=$ es\&nrm=iso>

24 Margaret Coffey, Lindsey Dugdill. Policies alone are not enough: Workplace health development in the public private and voluntary sectors. Critical Public Health. (on line) 2006; vol.16 n. ${ }^{\circ}$ 3. pp. 233-243. Disponible en la world wide web: http://www.bases.unal.edu.co:2108/ehost/pdfviewer/pdfviewer?vid=7\&hid=9\&sid=3bf1b68353f2-4950-91fa-02699e612f5f\%40sessionmgr11

25 Colombia - Congreso de la República. Ley 1122 (9 enero 2007). Por la cual se hacen algunas modificaciones en el Sistema General de Seguridad Social en Salud y se dictan otras disposiciones. Diario Oficial, Bogotá D.C., 2007. No. 46506. pp. 1-40

26 Ministerio de la Protección Social de Colombia. Resolución 1043 (3 abril 2006). Por la cual se establecen las condiciones que deben cumplir los Prestadores de Servicios de Salud para habilitar sus servicios e implementar el componente de auditoría para el mejoramiento de la calidad de la atención. Bogotá, D.C.: El Ministerio. $5 \mathrm{p}$

27 Ministerio de la Protección Social de Colombia. Resolución 1445 (8 mayo 2006). Por la cual se definen las funciones de la Entidad Acreditadora y se adoptan otras disposiciones. Bogotá, D.C.: El Ministerio. 2 p.

28 OPS-Ministerio de la Protección Social de Colombia. Relaciones Laborales en el sector salud. El Sistema General de Seguridad Social en Salud de Colombia: El caso de la ciudad de Cali. Julio, 2006.86 (p. 45) 
29 Ministerio de la Protección Social de Colombia y Universidad Javeriana. Recursos Humanos de la Salud en Colombia. Balance, competencias y prospectivas. 3. ${ }^{a}$ ed. Bogotá, 2008. 358 p.74

30 Bethania Ferreira, Maria Imaculada de Fátima Freitas. A implicação de trabalhadores de ambulatórios municipais, em Uberaba, Minas Gerais, Brasil, na reorganização de serviços preconizada pelo Sistema Único de Saúde. Cad. Saúde Pública [periódico na Internet]. 2008. 24(9). (citado 7 febrero 2010) pp. 2123-2130. Disponible en la world wide web: http://www.scielosp.org/scielo.php?script=sci_arttext\&pid=S0102311X2008000900018\&lng=en. doi: 10.1590/S0102-311X2008000900018

31 Luciana Barbosa de Oliveira, Eulalia Chaves-Maia. Saúde Psíquica dos Profissionais de Saúde em Hospitais Públicos. Revista de Salud Pública (on line), 2008 (citado noviembre 2009).vol. 10. n. ${ }^{\circ} 3$ pp. $405-413$. Disponible en la world wide web: <http://www.scielosp.org/scielo.php?script=sci_arttext\&pid=S0124$00642008000300005 \& \operatorname{lng}=\mathrm{en} \& \mathrm{nrm}=\mathrm{iso}>$

32 Alcaldía Distrital de Bogotá (on line). 2009. Disponible en la world wide web: www.samuelalcalde.com/ index.php?option=com_content\&view=article\&id=3834:salud\&catid=28:ultimas-hora

33 Organización Panamericana de la Salud. Taller Estrategia de promoción de la salud de los trabajadores. Relatoría. Op.cit.

34 Ministerio de la Protección Social de Colombia-Dirección General de Planeación y Análisis de Política. Informe de Actividades 2006-2007.

35 OMS. Quinta Conferencia Mundial de Promoción de la Salud. Documento técnico. Ciudad de México: Junio, 2000. p. 22. Disponible en: http://www.cepis.ops-oms.org/bvsdeps/fulltext/conf5.pdf

36 C, Dejours. A locura do trabalho. Estudo de psicopatología do trabalho. ed. 5. Ed. Sao Paulo. Atlas, 1994. p. 128.

37 Suzan Robroek, Frank Van Lenthe, Pepijn Van Empelen, Alex Burdorf. Determinants of participation in worksite health promotion programmes: a systematic review. International Journal of Behavioral Nutrition and Physical Activity (on line) 2009. vol.6. n. ${ }^{\circ}$ 26. (citado 20 enero de 2010). Disponible en la world wide web: http://www.ijbnpa.org/content/6/1/26

38 Riaño, Martha Isabel. Gestión de la seguridad y salud en el trabajo en hospitales públicos Bogotanos de alta complejidad: una perspectiva estratégica. Universidad Nacional de Colombia. 2009. p. 55

39 L. Renaud, Nathalie Kishchuk, Martin Juneau, Anil Nigam, Karine Tetreault, Marie Claude Leblanc. Implementation and outcomes of a comprehensive worksite health promotion program. Canadian Journal of Public Health (on line) 2008. vol. 99, N. ${ }^{\circ}$ 1, (citado 10 noviembre 2008) pp. 73-77. Disponible en la world wide web: http://www.bases.unal.edu.co:2108/ehost/pdfviewer/pdfviewer?vid=5\&hid=9\&sid=a51406eb1df2-4439-8441-8ca8cdbd20db\%40sessionmgr10

40 Alba Lucía Vélez Arango. Marco político y legal de la promoción de la salud. Disponible en la world wide web: http://colombiamedica.univalle.edu.co/VOL31NO2/promocion.htm

41 Organización Internacional del Trabajo. Trabajo decente en las Américas: una agenda hemisférica, 2006-2015. XVI Reunión Regional Americana. Brasilia, mayo de 2006. pp.13-45. Disponible en la World Wide Web: www.oit.org/public/spanish/standards/relm/rgmeet/16amr/dwork.pdf

42 European Network For Workplace Health Promotion. Luxembourg Declaration on Workplace Health Promotion in the European Union. Luxemburgo. 1997. 3 p. Disponible en la world wide web: http://www. enwhp.org/fileadmin/downloads/free/Luxembourg_Declaration_June2005_final.pdf. Consultado marzo de 2009.

43 Organización Mundial de la Salud. Carta de Bangkok para la promoción de la salud en un mundo globalizado. Disponible en la world wide web: http://www.who.int/healthpromotion/conferences/6gchp/BCHP_es.pdf

44 Organización Internacional del Trabajo. Convenio 187 sobre el marco promocional para la seguridad y salud en el trabajo, Adopción: 15:06:2006. Disponible en: http://www.ilo.org/ilolex/cgi-lex/convds.pl?C187

45 P. Buckle, P.J. Clarkson, R.Coleman, J.Ward, J. Anderson. Patient safety, systems design and ergonomics. Applied Ergonomics. [revista en Internet] 2006 julio [acceso 24 de agosto de 2008] 37 (4), [491-500] disponible en la world wide web: http://www.bases.unal.edu.co:2053/science?_ob=MImg\&_ imagekey= B6V1W4K48M9S-6-9\&_cdi=5685\&_user=1998314\&_pii=S $0003687006000548 \&$ \& orig =search \& coverDate=07\%2F31\%2F2006\&_sk=999629995\&view=c\&wchp=dGLzVlb-zSkzV\&md5= 30e2e84f75267fa4f6 3b05926e39e71d\&ie=/sdarticle.pdf

46 Amartya SEN. La democracia como valor universal. Journal of Democracy, 1999 vol. 10, n. ${ }^{\circ}$ 3, pp. 3-17, The John Hopkins University Press and National Endowment for Democracy. Disponible en la world wide web: http://www.docstoc.com/docs/3271077/La-democracia-como-valor-universal-Amartya-Sen-MAPAMUNDITOMADO-DE 
47 Joanne Sulman, Marylin Kanee, Paulette Stewart, Diane Savage. Does Difference Matter? Diversity and Human Rights in a Hospital Workplace. Soc Work Health Care. (on line) 2007; Vol.44. n. ${ }^{\circ}$ 3. (citado 6 abril 2010) pp. 145-59. Disponible en la world wide web: www.bases.unal.edu.co:2109/login.aspx?direct=true\&d $\mathrm{b}=\mathrm{mnh} \& \mathrm{AN}=17548272 \&$ lang $=$ es\&site $=$ ehost-live

48 Eklöf M, Ingelgard A, Hagberg M. Is participative ergonomics associated with better working environment and health? A study among Swedish white-collar VDU users. International Journal of Industrial Ergonomics (on line) 2004 vol. 34. n. ${ }^{\circ} 5$ (citado 15 noviembre 2008) pp.355-366 Disponible en la world wide web: http://www.bases.unal.edu.co:2053/science?_ob=ArticleListURL

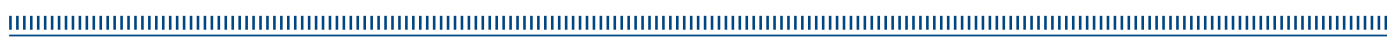

